www.jmscr.igmpublication.org

Impact Factor (SJIF): 6.379

Index Copernicus Value: 71.58

ISSN (e)-2347-176x ISSN (p) 2455-0450

crossref DOI: _https://dx.doi.org/10.18535/jmscr/v6i3.17

Journal Of Medical Science And Clinical Research

\title{
Efficacy and Safety of Addition of Teneligliptin to Type 2 Diabetes Patients Inadequately Controlled with Triple Drug Treatment with Glimepride + Metformin + Voglibose
}

\author{
Author \\ Dr SR Patnaik* \\ Associate Professor, Dept. Of Endocrinology, MKCG Medical College, Berhampur \\ *Corresponding Author \\ Dr SR Patnaik
}

\begin{abstract}
Type 2 diabetes is a significant health problem and its prevalence is increasing globally. There are several challenges to the management of type 2 diabetes in the Indian population. A single-center study evaluated the efficacy and safety of addition of teneligliptin $20 \mathrm{mg} /$ day to type 2 diabetes patients inadequately controlled with triple drug treatment with glimepride + metformin + voglibose. Overall, 81 were prescribed teneligliptin in addition to triple drug treatment. At study end, $62.12 \%$ patients achieved HbAlc levels $\leq 7 \%$. Patients reported only mild/transient adverse effects during the study period. It was concluded that addition of teneligliptin to triple drug therapy with glimepride + metformin + voglibose is efficacious to attain $\mathrm{HbAlc}$ below $7 \%$ in diabetes patients inadequately controlled with the triple therapy. It is also a safe drug since the adverse effects reported following the addition of teneligliptin were mild/transient.

Keywords: Teneligliptin, type 2 diabetes, triple drug therapy.
\end{abstract}

\section{Introduction}

Type 2 diabetes is a significant health problem with an increasing worldwide prevalence. ${ }^{1}$ There were more than 72 million cases of diabetes in India in $2017 .^{2}$ The prevalence of diabetes has been rising in India, not only in urban areas but in rural areas too. ${ }^{3}$

The management of type 2 diabetes is relatively challenging. Most international guidelines recommend an individualized management approach and an optimal HbA1c target tailored to every patient. ${ }^{1}$ To strike a balance, it is mandatory to attain optimal control of blood sugar levels and prevent microvascular events, and severe hypoglycemia. Cost, efficacy, potential side effects of the drugs, impact on body weight, comorbidities, and patient preferences also have significant roles to play in diabetes management. ${ }^{1}$

In a country like India, medical professionals face some major challenges in the prevention and management $\mathrm{f}$ type 2 diabetes. Some of these include increasing prevalence in both urban and rural areas; high prevalence of pre diabetes; genetic and environmental risk factors; delay in diagnosis; low levels of awareness among people; limited resources and health care facilities; high cost; suboptimal control of diabetes; and increased complications. ${ }^{3}$ 
All major guidelines recommend metformin as the first-line treatment in patients with diabetes unless contraindicated and tolerated well. ${ }^{1}$ The American Diabetes Association recommends that dual therapy should be considered in patients with newly diagnosed type 2 diabetes who have A1C $\geq 9 \%$. Among patients without atherosclerotic cardiovascular disease (ASCVD), if monotherapy or dual therapy fails to achieve or maintain the A1C goal over 3 months, an additional antihyperglycemic agent can be added based on drug-specific and patient factors. In those with established ASCVD, management should begin with lifestyle management and metformin and then a drug known to reduce major adverse cardiovascular events and cardiovascular mortality can be added after giving due consideration to drug-specific and patient factors.4 It is important to reevaluate the medication regimen and adjust it as needed. 4 When dual therapy fails to control blood glucose, a third agent can be added to the treatment regimen. ${ }^{1}$

If A1C target is not achieved after about 3 months on triple therapy, The ADA recommends combination injectable therapy.4 However, there may be situations where insulin and other injectables are not an option.

This study thus sought to evaluate the effect of adding an oral hypoglycemic agent, teneligliptin, to a triple drug therapy regimen in patients not adequately controlled with the triple therapy.

\section{Objective}

The study was conducted to evaluate the efficacy and safety of addition of teneligliptin to type 2 diabetes patients inadequately controlled with triple drug treatment with glimepride + metformin + voglibose.

\section{Patients and Methods}

This was a single-center study that included 81 patients with type 2 diabetes whose blood sugar levels were not adequately controlled on triple drug therapy with glimepride + metformin + voglibose. All the subjects were prescribed teneligliptin $20 \mathrm{mg}$ OD in addition to triple drug treatment. The patients were studied for a period of 3 months.

Patients with a significant complication were not included in the study. Fifteen patients dropped out from the study. Of these, 4 developed significant osmotic symptoms, 2 had acute myocardial infarction, 2 patients had a cerebrovascular accident, 1 developed urinary tract infection (UTI), 2 had pneumonia and 4 patients were lost to follow-up. Therefore, data was available for 66 patients.

All patients underwent blood sugar evaluation before, 1 month, 2 months and 3 months after the addition of teneligliptin. HbA1c levels were evaluated before and 3 months after teneligliptin addition.

The demographic characteristics of the patients enrolled in the study are summarized in table 1. The study population was in the age range of 3870 years with 40 males and 26 females.

Table 1. Demographic characteristics of study population

\begin{tabular}{|l|c|c|c|c|c|c|c|c|c|c|c|}
\hline Sex & Age & $\begin{array}{c}\text { Height } \\
(\mathbf{c m})\end{array}$ & $\begin{array}{c}\text { Weight } \\
(\mathbf{k g})\end{array}$ & $\begin{array}{c}\text { Duration } \\
\text { of DM }\end{array}$ & UR & CR & TC & TG & LD & HD & VD \\
\hline M & 46 & 166 & 64 & 3 YRS & 36 & 0.8 & 208 & 244 & 124 & 36 & 48 \\
\hline M & 63 & 164 & 72 & 2 YRS & 24 & 1.0 & 267 & 174 & 195 & 40 & 32 \\
\hline M & 52 & 170 & 80 & 2 YRS & 30 & 1.3 & 204 & 160 & 120 & 52 & 32 \\
\hline M & 61 & 144 & 59 & 1YRS & 24 & 0.8 & 168 & 84 & 116 & 36 & 16 \\
\hline F & 70 & 158 & 62 & 3 YRS & 31 & 1.2 & 184 & 120 & 124 & 40 & 24 \\
\hline F & 62 & 154 & 48 & 2 YRS & 26 & 1.0 & 190 & 140 & 120 & 35 & 35 \\
\hline M & 47 & 165 & 46 & 2 YRS & 16 & 0.6 & 154 & 174 & 79 & 40 & 35 \\
\hline F & 50 & 162 & 50 & 4 YRS & 20 & 1.0 & 162 & 150 & 90 & 42 & 30 \\
\hline M & 60 & 163 & 54 & 3 YRS & 18 & 0.8 & 140 & 110 & 77 & 41 & 22 \\
\hline F & 64 & 170 & 64 & 4 YRS & 34 & 12 & 220 & 160 & 138 & 50 & 32 \\
\hline
\end{tabular}




\begin{tabular}{|c|c|c|c|c|c|c|c|c|c|c|c|}
\hline $\mathrm{F}$ & 47 & 154 & 50 & 2 YRS & 28 & 08 & 176 & 144 & 94 & 54 & 28 \\
\hline $\mathrm{M}$ & 47 & 166 & 60 & 1 YRS & 30 & 1.1 & 217 & 190 & 119 & 60 & 38 \\
\hline $\mathrm{M}$ & 50 & 144 & 57 & 2 YRS & 18 & 0.7 & 142 & 112 & 79 & 41 & 22 \\
\hline$M$ & 54 & 169 & 72 & $1 \mathrm{YR}$ & 30 & 1.2 & 224 & 210 & 120 & 62 & 42 \\
\hline $\mathrm{M}$ & 52 & 174 & 70 & $1 \mathrm{YR}$ & 26 & 1.0 & 250 & 208 & 151 & 58 & 41 \\
\hline $\mathrm{M}$ & 60 & 172 & 74 & 2 YRS & 31 & 1.1 & 301 & 400 & 27 & 50 & 80 \\
\hline $\mathrm{F}$ & 48 & 160 & 56 & 3 YRS & 26 & 1.0 & 170 & 142 & 98 & 44 & 28 \\
\hline $\mathrm{F}$ & 54 & 154 & 50 & 3 YRS & 22 & 1.1 & 184 & 136 & 107 & 50 & 27 \\
\hline $\mathrm{F}$ & 54 & 160 & 54 & 2 YRS & 18 & 0.8 & 210 & 174 & 118 & 58 & 34 \\
\hline$M$ & 48 & 164 & 60 & 4 YRS & 24 & 1.1 & 240 & 200 & 146 & 54 & 40 \\
\hline $\mathrm{F}$ & 42 & 154 & 58 & $1 \mathrm{YR}$ & 18 & 0.8 & 161 & 140 & 92 & 41 & 28 \\
\hline $\mathrm{F}$ & 58 & 161 & 60 & 3 YRS & 28 & 1.1 & 220 & 160 & 125 & 63 & 32 \\
\hline $\mathrm{M}$ & 44 & 164 & 62 & $1 \mathrm{YR}$ & 20 & 1.1 & 202 & 170 & 114 & 54 & 34 \\
\hline $\mathrm{F}$ & 38 & 157 & 44 & $1 \mathrm{YR}$ & 16 & 0.8 & 174 & 146 & 104 & 41 & 29 \\
\hline $\bar{M}$ & 65 & 174 & 68 & 2 YRS & 20 & 1.2 & 217 & 154 & 125 & 62 & 30 \\
\hline $\mathrm{M}$ & 70 & 169 & 70 & $1 \mathrm{YR}$ & 21 & 1.1 & 273 & 180 & 163 & 74 & 36 \\
\hline $\mathrm{M}$ & 64 & 166 & 65 & $1 \mathrm{YR}$ & 14 & 1.0 & 172 & 144 & 83 & 61 & 28 \\
\hline $\mathrm{M}$ & 48 & 160 & 68 & 2 YRS & 22 & 1.0 & 188 & 151 & 107 & 51 & 30 \\
\hline $\mathrm{M}$ & 50 & 168 & 70 & $1 \mathrm{YR}$ & 30 & 1.0 & 264 & 500 & & 81 & \\
\hline $\mathrm{F}$ & 48 & 170 & 71 & 6 YRS & 36 & 1.4 & 300 & 190 & 182 & 70 & 38 \\
\hline $\mathrm{F}$ & 60 & 161 & 56 & 2 YRS & 24 & 0.8 & 202 & 200 & 110 & 52 & 40 \\
\hline $\mathrm{F}$ & 56 & 158 & 53 & $1 \mathrm{YR}$ & 20 & 1.1 & 246 & 200 & 140 & 60 & 40 \\
\hline$M$ & 42 & 156 & 64 & $1 \mathrm{YR}$ & 30 & 1.2 & 300 & 200 & 203 & 57 & 40 \\
\hline $\mathrm{M}$ & 52 & 162 & 66 & 5 YRS & 31 & 1.4 & 267 & 304 & 142 & 64 & 61 \\
\hline $\mathrm{M}$ & 64 & 170 & 70 & 3 YRS & 28 & 1.3 & 220 & 170 & 125 & 61 & 34 \\
\hline $\mathrm{M}$ & 54 & 168 & 68 & $1 \mathrm{YR}$ & 22 & 1.2 & 194 & 94 & 112 & 64 & 18 \\
\hline
\end{tabular}

\begin{tabular}{|c|c|c|c|c|c|c|c|c|c|c|c|}
\hline Sex & Age & $\begin{array}{l}\text { Height } \\
\text { (cm) }\end{array}$ & $\begin{array}{c}\text { Weight } \\
\text { (kg) }\end{array}$ & $\begin{array}{c}\text { Duration } \\
\text { of DM }\end{array}$ & UR & CR & TC & TG & LD & HD & VD \\
\hline $\mathrm{F}$ & 60 & 154 & 52 & 4 YRS & 28 & 1.1 & 220 & 161 & 122 & 66 & 32 \\
\hline $\mathrm{M}$ & 63 & 164 & 64 & 6 YRS & 24 & 1.0 & 360 & 400 & 122 & 60 & 8 \\
\hline $\mathrm{F}$ & 45 & 160 & 54 & 3 YRS & 20 & 1.1 & 216 & 188 & 121 & 58 & 37 \\
\hline $\mathrm{F}$ & 52 & 156 & 56 & 5 YRS & 18 & 0.8 & 176 & 200 & 66 & 60 & 50 \\
\hline M & 61 & 164 & 58 & 4 YRS & 24 & 1.2 & 241 & 216 & 132 & 66 & 43 \\
\hline $\mathrm{F}$ & 56 & 158 & 62 & 3 YRS & 29 & 1.4 & 196 & 201 & 99 & 56 & 41 \\
\hline $\mathrm{F}$ & 60 & 161 & 63 & 4 YRS & 30 & 0.9 & 184 & 214 & 84 & 58 & 42 \\
\hline $\mathrm{F}$ & 61 & 164 & 59 & 4 YRS & 26 & 1.1 & 222 & 166 & 128 & 61 & 33 \\
\hline M & 62 & 163 & 60 & 5 YRS & 17 & 0.8 & 264 & 206 & 164 & 59 & 41 \\
\hline $\mathrm{M}$ & 63 & 163 & 69 & 3 YRS & 27 & 1.1 & 361 & 256 & 230 & 80 & 51 \\
\hline $\mathrm{M}$ & 59 & 166 & 68 & 4 YRS & 33 & 1.3 & 294 & 186 & 191 & 66 & 37 \\
\hline M & 59 & 158 & 60 & 4 YRS & 26 & 1.3 & 192 & 180 & 96 & 60 & 36 \\
\hline $\bar{F}$ & 61 & 162 & 62 & 5 YRS & 34 & 1.2 & 204 & 200 & 100 & 64 & 40 \\
\hline $\mathrm{F}$ & 57 & 158 & 58 & 6 YRS & 28 & 1.3 & 210 & 254 & 100 & 60 & 50 \\
\hline $\mathrm{F}$ & 56 & 160 & 64 & 4 YRS & 24 & 1.0 & 186 & 201 & 98 & 48 & 40 \\
\hline M & 60 & 157 & 60 & 4 YRS & 20 & 0.8 & 312 & 214 & 207 & 63 & 42 \\
\hline $\mathrm{M}$ & 70 & 166 & 70 & 5 YRS & 30 & 1.1 & 284 & 302 & 150 & 74 & 60 \\
\hline $\bar{M}$ & 66 & 170 & 68 & 3 YRS & 26 & 1.2 & 263 & 201 & 142 & 80 & 41 \\
\hline M & 60 & 186 & 70 & 2 YRS & 31 & 1.1 & 194 & 214 & 89 & 63 & 42 \\
\hline $\mathrm{M}$ & 56 & 168 & 68 & 3 YRS & 24 & 1.3 & 216 & 194 & 127 & 51 & 38 \\
\hline $\bar{F}$ & 46 & 163 & 60 & 2 YRS & 26 & 1.2 & 204 & 316 & 97 & 44 & 63 \\
\hline $\mathrm{F}$ & 42 & 164 & 60 & 3 YRS & 20 & 1.1 & 190 & 160 & 107 & 51 & 32 \\
\hline $\mathrm{M}$ & 68 & 166 & 61 & 3 YRS & 18 & 1.1 & 261 & 240 & 142 & 61 & 58 \\
\hline $\bar{M}$ & 70 & 168 & 62 & 2 YRS & 22 & 0.9 & 201 & 196 & 108 & 54 & 39 \\
\hline $\mathrm{M}$ & 56 & 162 & 59 & 6 YRS & 20 & 1.3 & 309 & 257 & 208 & 50 & 51 \\
\hline $\mathrm{M}$ & 63 & 166 & 63 & 9 YRS & 24 & 1.2 & 244 & 210 & 139 & 63 & 42 \\
\hline M & 49 & 168 & 70 & $10 \mathrm{YRS}$ & 17 & 0.9 & 314 & 307 & 193 & 60 & 61 \\
\hline $\mathrm{F}$ & 58 & 163 & 60 & 12 YRS & 34 & 1.1 & 304 & 264 & 171 & 71 & 52 \\
\hline $\mathrm{M}$ & 60 & 161 & 64 & 2 YRS & 27 & 1.0 & 210 & 125 & 115 & 60 & 35 \\
\hline M & 67 & 167 & 84 & 4 YRS & 30 & 1.2 & 304 & 500 & & 50 & \\
\hline
\end{tabular}




\section{Results}

It was observed that at the end of the study period, 62.12\% (n=41) patients attained HbA1c levels $\leq 7 \%$. There were only mild/transient adverse effects in the patients, with no drop outs due to an adverse reaction. About $4 \%$ patients developed a UTI and 6\% developed an upper respiratory tract infection (URTI) during the course of the study. No death was reported during the study.

Table 2 summarizes the blood sugar levels before teneligliptin and 1-, 2-, and 3-months after teneligliptin treatment. It also depicts the $\mathrm{HbA} 1 \mathrm{c}$ levels at the start and end of study period.

Table 2 Blood sugar and $\mathrm{HbA} 1 \mathrm{c}$ levels before and after teneligliptin treatment

\begin{tabular}{|c|c|c|c|c|c|c|c|c|c|c|}
\hline \multirow[t]{2}{*}{ Sl. No. } & \multicolumn{2}{|c|}{$\begin{array}{l}\text { Blood sugar } \\
\text { before } \\
\text { teneligliptin } \\
\text { addition }\end{array}$} & \multicolumn{2}{|c|}{$\begin{array}{c}\text { Blood sugar } 1 \text { month } \\
\text { after teneligliptin } \\
\text { addition }\end{array}$} & \multicolumn{2}{|c|}{$\begin{array}{c}\text { Blood sugar } 2 \\
\text { months after } \\
\text { teneligliptin } \\
\text { addition }\end{array}$} & \multicolumn{2}{|c|}{$\begin{array}{c}\text { Blood sugar } 3 \\
\text { months after } \\
\text { teneligliptin } \\
\text { addition } \\
\end{array}$} & \multicolumn{2}{|c|}{ HbA1c } \\
\hline & $\mathbf{F}$ & $\mathbf{P P}$ & $\mathbf{F}$ & PP & $\mathbf{F}$ & $\mathbf{P P}$ & $\mathbf{F}$ & $\mathbf{P P}$ & $\begin{array}{l}\text { Before } \\
\text { study }\end{array}$ & $\begin{array}{l}\text { After } \\
\text { study }\end{array}$ \\
\hline 1 & 216 & 317 & 160 & 195 & 130 & 190 & 114 & 170 & 7.6 & 6.8 \\
\hline 2 & 250 & 380 & 150 & 210 & 140 & 194 & 130 & 176 & 10.2 & 7.1 \\
\hline 3 & 175 & 287 & 160 & 202 & 142 & 180 & 124 & 166 & 9.1 & 7.0 \\
\hline 4 & 163 & 306 & 130 & 260 & 126 & 200 & 110 & 180 & 8.7 & 6.8 \\
\hline 5 & 140 & 284 & 110 & 204 & 114 & 180 & 120 & 161 & 7.6 & 6.6 \\
\hline 6 & 201 & 314 & 170 & 301 & 120 & 204 & 114 & 180 & 9.4 & 6.8 \\
\hline 7 & 190 & 293 & 140 & 209 & 134 & 170 & 140 & 160 & 9.6 & 7.0 \\
\hline 8 & 154 & 275 & 134 & 175 & 126 & 170 & 110 & 180 & 7.7 & 6.7 \\
\hline 9 & 170 & 297 & 120 & 214 & 124 & 180 & 114 & 174 & 7.4 & 6.6 \\
\hline 10 & 204 & 317 & 190 & 287 & 184 & 304 & 214 & 314 & 9.4 & 9.0 \\
\hline 11 & 190 & 320 & 160 & 260 & 144 & 170 & 130 & 174 & 8.3 & 6.7 \\
\hline 12 & 184 & 290 & 161 & 210 & 130 & 180 & 126 & 160 & 8.1 & 6.9 \\
\hline 13 & 198 & 303 & 154 & 190 & 120 & 174 & 124 & 180 & 8.4 & 7.0 \\
\hline 14 & 204 & 354 & 160 & 210 & 144 & 190 & 120 & 164 & 9.1 & 6.9 \\
\hline 15 & 176 & 307 & 164 & 268 & 137 & 204 & 124 & 180 & 9.4 & 7.3 \\
\hline 16 & 184 & 403 & 190 & 368 & 170 & 344 & 154 & 302 & 10.3 & 9.7 \\
\hline 17 & 160 & 290 & 130 & 240 & 120 & 170 & 110 & 168 & 8.4 & 6.7 \\
\hline 18 & 110 & 260 & 90 & 140 & 94 & 162 & 101 & 170 & 7.7 & 6.6 \\
\hline 19 & 216 & 376 & 170 & 340 & 160 & 310 & 166 & 300 & 11.4 & 9.4 \\
\hline 20 & 170 & 266 & 140 & 204 & 130 & 170 & 124 & 174 & 7.6 & 6.8 \\
\hline 21 & 204 & 344 & 190 & 284 & 176 & 270 & 160 & 284 & 10.1 & 9.3 \\
\hline 22 & 191 & 250 & 130 & 164 & 110 & 170 & 124 & 180 & 8.0 & 7.4 \\
\hline 23 & 170 & 274 & 160 & 217 & 140 & 240 & 144 & 230 & 7.6 & 7.4 \\
\hline 24 & 210 & 294 & 154 & 198 & 130 & 170 & 114 & 180 & 8.3 & 7.1 \\
\hline 25 & 140 & 281 & 130 & 204 & 126 & 177 & 120 & 164 & 7.4 & 6.6 \\
\hline 26 & 201 & 377 & 154 & 317 & 124 & 170 & 117 & 168 & 10.1 & 7.4 \\
\hline 27 & 214 & 356 & 190 & 284 & 194 & 270 & 201 & 264 & 11.1 & 9.8 \\
\hline 28 & 194 & 314 & 120 & 219 & 116 & 170 & 110 & 174 & 9.3 & 7.3 \\
\hline 29 & 174 & 287 & 124 & 194 & 114 & 180 & 112 & 163 & 8.0 & 7.0 \\
\hline 30 & 168 & 301 & 151 & 237 & 130 & 201 & 114 & 190 & 8.1 & 7.2 \\
\hline 31 & 191 & 317 & 174 & 284 & 160 & 231 & 140 & 212 & 8.3 & 7.9 \\
\hline 32 & 201 & 311 & 140 & 174 & 114 & 164 & 94 & 170 & 8.1 & 6.8 \\
\hline 32 & 166 & 302 & 130 & 214 & 114 & 170 & 92 & 160 & 83 & 6.9 \\
\hline 33 & 160 & 264 & 110 & 170 & 99 & 160 & 84 & 141 & 7.8 & 6.4 \\
\hline 34 & 190 & 287 & 104 & 180 & 101 & 154 & 93 & 144 & 8.1 & 6.3 \\
\hline 35 & 210 & 367 & 121 & 190 & 117 & 170 & 101 & 163 & 9.1 & 7.0 \\
\hline 36 & 180 & 271 & 140 & 230 & 116 & 191 & 117 & 174 & 9.4 & 6.9 \\
\hline 37 & 174 & 301 & 160 & 276 & 154 & 290 & 160 & 280 & 9.0 & 8.6 \\
\hline 38 & 190 & 341 & 130 & 241 & 134 & 206 & 130 & 184 & 9.2 & 7.2 \\
\hline 39 & 163 & 257 & 124 & 191 & 107 & 184 & 94 & 163 & 8.7 & 7.0 \\
\hline 40 & 154 & 240 & 114 & 184 & 90 & 170 & 86 & 154 & 7.4 & 6.6 \\
\hline 41 & 184 & 257 & 130 & 203 & 110 & 174 & 101 & 167 & 7.6 & 6.9 \\
\hline
\end{tabular}




\begin{tabular}{|l|c|c|c|c|c|c|c|c|c|c|}
\hline 42 & 184 & 314 & 140 & 190 & 104 & 174 & 84 & 170 & 9.1 & 7.3 \\
\hline 43 & 190 & 344 & 174 & 274 & 160 & 284 & 154 & 270 & 11.1 & 9.3 \\
\hline 44 & 163 & 271 & 120 & 164 & 114 & 170 & 94 & 154 & 8.4 & 6.9 \\
\hline 45 & 172 & 244 & 124 & 190 & 110 & 164 & 97 & 154 & 7.4 & 6.6 \\
\hline 46 & 191 & 287 & 144 & 214 & 104 & 170 & 90 & 157 & 8.1 & 6.7 \\
\hline 47 & 170 & 301 & 130 & 190 & 101 & 164 & 84 & 161 & 8.2 & 6.6 \\
\hline 48 & 201 & 377 & 194 & 354 & 200 & 334 & 170 & 300 & 11.7 & 10.4 \\
\hline 49 & 206 & 204 & 134 & 180 & 120 & 176 & 110 & 170 & 9.4 & 7.3 \\
\hline 50 & 170 & 301 & 160 & 214 & 154 & 197 & 184 & 211 & 8.7 & 8.5 \\
\hline 51 & 160 & 271 & 130 & 197 & 124 & 161 & 110 & 154 & 7.8 & 6.9 \\
\hline 52 & 154 & 244 & 110 & 184 & 90 & 170 & 93 & 168 & 7.7 & 6.5 \\
\hline 53 & 150 & 270 & 108 & 191 & 97 & 175 & 101 & 171 & 7.7 & 6.8 \\
\hline 54 & 190 & 317 & 114 & 214 & 104 & 190 & 106 & 201 & 8.1 & 7.4 \\
\hline 55 & 201 & 314 & 140 & 200 & 136 & 180 & 126 & 174 & 8.4 & 7.3 \\
\hline 56 & 164 & 281 & 121 & 174 & 114 & 161 & 90 & 154 & 7.6 & 6.7 \\
\hline 57 & 166 & 301 & 101 & 201 & 96 & 174 & 97 & 163 & 8.1 & 7.1 \\
\hline 58 & 141 & 222 & 90 & 174 & 92 & 190 & 84 & 174 & 7.4 & 6.9 \\
\hline 59 & 161 & 284 & 99 & 163 & 92 & 166 & 87 & 154 & 7.5 & 6.7 \\
\hline 60 & 184 & 314 & 114 & 184 & 110 & 178 & 90 & 174 & 8.3 & 6.8 \\
\hline 61 & 140 & 281 & 104 & 170 & 90 & 166 & 88 & 160 & 7.6 & 6.4 \\
\hline 62 & 210 & 344 & 170 & 302 & 166 & 312 & 154 & 301 & 9.7 & 9.3 \\
\hline 63 & 160 & 270 & 106 & 184 & 94 & 172 & 90 & 166 & 7.7 & 6.6 \\
\hline 64 & 174 & 284 & 121 & 193 & 91 & 174 & 84 & 170 & 7.9 & 6.4 \\
\hline 65 & 145 & 230 & 130 & 190 & 126 & 184 & 127 & 190 & 7.3 & 6.9 \\
\hline 66 & 150 & 274 & 94 & 204 & 96 & 190 & 94 & 199 & 7.4 & 7.1 \\
\hline
\end{tabular}

\section{Discussion}

Quadruple therapy regimen is a possible approach in the management of type 2 diabetes, ${ }^{5}$ and warrants research. In this study, a fourth oral drug was added to triple oral therapy in patients with inadequately controlled diabetes mellitus. Teneligliptin $20 \mathrm{mg}$ OD was added to the triple drug therapy regimen in the patients and the patients were analyzed for 3 months. At the end of the third month, nearly $62 \%$ of the patients attained an $\mathrm{HbA} 1 \mathrm{c}$ of $\leq 7 \%$, which is quite encouraging. The adverse effects were only mild and the drug was well tolerated overall.

Teneligliptin is a relatively new oral dipeptidyl peptidase 4 (DPP-4) inhibitor employed for the management of type 2 diabetes mellitus. Published literature highlights the efficacy of teneligliptin as monotherapy and in combination with other oral agents, and insulin in short-term and long-term studies. Teneligliptin has been shown to yield a reduction in HbA1c of $0.8 \%-$ $0.9 \%$ within 12 weeks of treatment. This is a safe and well-tolerated drug. It is also safe to be used in patients with mild, moderate, or severe renal impairment or end-stage renal disease without the need for any dose adjustment. Patients with mildto-moderate hepatic impairment can also be prescribed teneligliptin. ${ }^{6}$

Eto et $\mathrm{al}^{7}$ assessed blood glucose control over $24 \mathrm{~h}$ and the safety of teneligliptin 10 and $20 \mathrm{mg}$ in patients with type 2 diabetes mellitus inadequately controlled with diet and exercise. Patients given teneligliptin had significantly lower 2-h postprandial glucose (2-h PPG), 24-h mean glucose and fasting plasma glucose levels as compared to placebo group. No hypoglycemic symptoms or serious adverse events were seen in the patients. In a study by Kadowaki and Kondo, ${ }^{8}$ treatment with teneligliptin for 12 weeks was found to yield significant and clinically meaningful reductions in $\mathrm{HbA1c}$ and FPG.

Teneligliptin has been found to be effective in combination therapy as well. Kim et $\mathrm{al}^{9}$ determined the efficacy and safety of teneligliptin in combination with metformin in type 2 diabetes patients inadequately controlled with metformin monotherapy. The differences between the teneligliptin and placebo groups for changes in $\mathrm{HbA1c}$ and fasting plasma glucose levels were $0.78 \%$ and $-1.24 \quad \mathrm{mmol} / \mathrm{l} \quad(22.42 \mathrm{mg} / \mathrm{dl})$, 
respectively, at week 16 . The addition of teneligliptin once daily to metformin was effective and well tolerated.

Considering the efficacy of teneligliptin in the management of diabetes, I decided to include this drug as the fourth add-on oral hypoglycemic agent in my diabetes patients not controlled well on triple therapy and found the drug to yield positive outcomes. The drug was effective in attaining target HbA1c level in a large number of the study patients and was well-tolerated.

Therefore, teneligliptin could well be the choice of fourth drug added to a triple therapy regimen in patients who cannot be given insulin treatment, in order to achieve the desired HbA1c target.

\section{Conclusion}

Addition of teneligliptin to type 2 diabetes patients inadequately controlled with triple drug treatment with glimepride + metformin + voglibose is safe and effective. This regimen led to the achievement of $\mathrm{HbAlc} \leq 7 \%$ in about $62.12 \%$ patients.

\section{References}

1. Downes MJ, Bettington EK, Gunton JE, Turkstra E. Triple therapy in type 2 diabetes; a systematic review and network meta-analysis. PeerJ. 2015; 3: e1461.

2. Available from: https://www.idf.org/ournetwork/regions-members/south-eastasia/members/94-india.html. Accessed on February 23, 2018.

3. Ramachandran A, Shetty AS, Nanditha A, Snehalatha C. Type 2 Diabetes in India: Challenges and Possible Solutions. Available from: http://www.apiindia.org/medicine_update_ 2013/chap40.pdf. Accessed on February 23, 2018.

4. Pharmacologic Approaches to Glycemic Treatment: Standards of Medical Care in Diabetes-2018. Diabetes Care 2018 Jan; 41(Supplement 1): S73-S85.
5. Torre EM, Tejedor JL, Menendez SA, et al. Recommendations for the pharmacologic treatment of hyperglycemia in type 2 diabetes. Consensus document. Nefrologia (English Version) 2011;31:1726.

6. Sharma SK, Panneerselvam A, Singh KP, et al. Teneligliptin in management of type 2 diabetes mellitus. Diabetes Metab Syndr Obes. 2016; 9: 251-260.

7. Eto $\mathrm{T}$, Inoue $\mathrm{S}$, Kadowaki $\mathrm{T}$. Effects of once-daily teneligliptin on 24-h blood glucose control and safety in Japanese patients with type 2 diabetes mellitus: a 4week, randomized, double-blind, placebocontrolled trial. Diabetes Obes Metab. 2012 Nov;14(11):1040-6.

8. Kadowaki T, Kondo K. Efficacy, safety and dose-response relationship of teneligliptin, a dipeptidyl peptidase-4 inhibitor, in Japanese patients with type 2 diabetes mellitus. Diabetes Obes Metab. 2013 Sep;15(9):810-8.

9. Kim MK, Rhee EJ, Han KA, et al. Efficacy and safety of teneligliptin, a dipeptidyl peptidase-4 inhibitor, combined with metformin in Korean patients with type 2 diabetes mellitus: a 16-week, randomized, double-blind, placebocontrolled phase III trial. Diabetes Obes Metab. 2015 Mar;17(3):309-12. 